\title{
Accumulation of sequence variants in genes of Wnt signaling and focal adhesion pathways in human corneas further explains their involvement in keratoconus
}

\author{
Justyna A Karolak $^{1,2}$, Tomasz Gambin ${ }^{3}$, Malgorzata Rydzanicz ${ }^{4}$, Piotr Polakowski ${ }^{5}$, Rafal Ploski ${ }^{4}$, Jacek P Szaflik ${ }^{5}$ \\ , Marzena Gajecka ${ }^{\text {Corresp. 1,2 }}$ \\ ${ }^{1}$ Chair and Department of Genetics and Pharmaceutical Microbiology, Poznan University of Medical Sciences, Poznan, Poland \\ Institute of Human Genetics, Polish Academy of Sciences, Poznan, Poland \\ 3 Institute of Computer Science, Warsaw University of Technology, Warsaw, Poland \\ 4 Department of Medical Genetics, Medical University of Warsaw, Warsaw, Poland \\ 5 Department of Ophthalmology, Medical University of Warsaw, Warsaw, Poland \\ Corresponding Author: Marzena Gajecka \\ Email address: gamar@man.poznan.pl
}

Background. Keratoconus (KTCN) is a protrusion and thinning of the cornea, resulting in loss of visual acuity. The etiology of KTCN remains unclear. The purpose of this study was to assess the potential involvement of new genetic variants in KTCN etiology based on both the genomic and transcriptomic findings recognized in the same corneal tissues.

Methods. Corneal tissues derived from five unrelated Polish individuals with KTCN were examined using exome sequencing (ES), followed by enrichment analyses. For comparison purposes, the datasets comprising ES data of five randomly selected Polish individuals without ocular abnormalities and five Polish patients with high myopia (HM) were used. Expression levels of selected genes from the overrepresented pathways were obtained from the previous RNA-Seq study.

Results. Exome capture discovered 117 potentially relevant variants that were further narrowed by gene overrepresentation analyses. In each of five patients, the assessment of functional interactions revealed rare (MAF $\leq 0.01$ ) DNA variants in at least one gene from Wnt signaling (VANGL1, WNT1, PPP3CC, LRP6, FZD2) and focal adhesion (BIRC2, PAK6, COL4A4, PPP1R12A, PTK6) pathways. No genes involved in pathways enriched in KTCN corneas were overrepresented in our control sample sets.

Conclusions. The results of this first pilot ES profiling of human KTCN corneas emphasized that accumulation of sequence variants in several genes from Wnt signaling and/or focal adhesion pathways might cause the phenotypic effect and further points to a complex etiology of KTCN. 
1 Accumulation of sequence variants in genes of Wnt signaling and focal adhesion pathways

2 in human corneas further explains their involvement in keratoconus

3

4 Justyna A. Karolak ${ }^{1,2}$, Tomasz Gambin ${ }^{3}$, Malgorzata Rydzanicz ${ }^{4}$, Piotr Polakowski ${ }^{5}$, Rafal

5 Ploski ${ }^{4}$, Jacek P. Szaflik ${ }^{5}$, Marzena Gajecka ${ }^{1,2}$

$6{ }^{1}$ Chair and Department of Genetics and Pharmaceutical Microbiology, Poznan University of

7 Medical Sciences, Poznan, Poland;

$8 \quad{ }^{2}$ Institute of Human Genetics, Polish Academy of Sciences, Poznan, Poland;

$9 \quad{ }^{3}$ Institute of Computer Science, Warsaw University of Technology, Warsaw, Poland;

$10{ }^{4}$ Department of Medical Genetics, Medical University of Warsaw, Warsaw, Poland;

$11{ }^{5}$ Department of Ophthalmology, Medical University of Warsaw, Warsaw, Poland.

13 Corresponding author

14 Marzena Gajecka ${ }^{1,2}$

15 Strzeszynska 32, Poznan, 60-479, Poland;

16 E-mail address: gamar@man.poznan.pl 
24 Abstract

25 Background. Keratoconus (KTCN) is a protrusion and thinning of the cornea, resulting in loss of visual acuity. The etiology of KTCN remains unclear. The purpose of this study was to assess the potential involvement of new genetic variants in KTCN etiology based on both the genomic and transcriptomic findings recognized in the same corneal tissues.

Methods. Corneal tissues derived from five unrelated Polish individuals with KTCN were examined using exome sequencing (ES), followed by enrichment analyses. For comparison purposes, the datasets comprising ES data of five randomly selected Polish individuals without ocular abnormalities and five Polish patients with high myopia (HM) were used. Expression levels of selected genes from the overrepresented pathways were obtained from the previous RNA-Seq study.

Results. Exome capture discovered 117 potentially relevant variants that were further narrowed by gene overrepresentation analyses. In each of five patients, the assessment of functional interactions revealed rare $(\mathrm{MAF} \leq 0.01) \mathrm{DNA}$ variants in at least one gene from Wnt signaling (VANGL1, WNT1, PPP3CC, LRP6, FZD2) and focal adhesion (BIRC2, PAK6, COL4A4, PPP1R12A, PTK6) pathways. No genes involved in pathways enriched in KTCN corneas were overrepresented in our control sample sets.

Conclusions. The results of this first pilot ES profiling of human KTCN corneas emphasized that accumulation of sequence variants in several genes from Wnt signaling and/or focal adhesion pathways might cause the phenotypic effect and further points to a complex etiology of $44 \mathrm{KTCN}$.

\section{Introduction}


Keratoconus (KTCN) is an eye disease characterized by progressive thinning and conical

48

49

50

51

52

53

54

protrusion of the cornea. ${ }^{1}$ The structural abnormalities in different layers of corneal tissue result in altered refractive powers and a loss of visual function. ${ }^{2}$ The first symptoms of KTCN usually appear during puberty or early in the third decade of life. ${ }^{2}$ The management of this condition depends on the disease state and includes visual correction by contact lenses, corneal collagen cross-linking, or corneal transplant surgery. ${ }^{3,4}$ The incidence of $\mathrm{KTCN}$ is one in 2,000 individuals in the general population. ${ }^{2}$ However, the incidence may vary depending on the geographic location and ethnicity of the studied population. The estimated incidence of KTCN is higher in Indians, Chinese, Pacific, and Maori ethnicities compared with Caucasians..$^{5-10}$ The environmental factors, such as eye rubbing or contact lens wear, influence disease development. ${ }^{11}$ However, genetic triggers also play an important role in KTCN. .12 Several genomic strategies have been implemented for finding candidate genes, including both simple molecular techniques and high-throughput technologies. ${ }^{1,12-16}$

Linkage studies, performed in KTCN families, have led to the identification of multiple chromosomal regions linked to KTCN, including two replicated loci at 5q. ${ }^{17-21}$ The genome-wide association studies (GWAS) have identified variants mapped near $H G F, C O L 5 A 1$, FOXO1, RAB3GAP1, or ZNF469, associated with KTCN risk. ${ }^{22-25}$ However, their contribution to KTCN needs to be clarified. Several candidate genes for KTCN have also been identified using Sanger sequencing or next generation sequencing (NGS) including VSX1, SOD1, and DOCK9 or SKP1, MPDZ, FLG, PPIP5K2, and PCSK1, respectively. ${ }^{26-32}$ However, variants detected in those genes were present in a small fraction of KTCN patients or particular populations only. Analysis of transcriptome profiles of human KTCN and non-KTCN corneas by a highthroughput RNA sequencing (RNA-Seq) showed the deregulation of numerous genes in KTCN 
corneas ${ }^{33}$ The significant downregulation was observed among genes in collagen synthesis and maturation pathways, as well as in the TGF- $\beta$, Hippo, and Wnt signaling pathways. ${ }^{33}$ The results of subsequent RNA studies further support the potential role of genes involved in the extracellular matrix, TGF- $\beta$, and Wnt molecular cascades in KTCN pathogenesis. ${ }^{34-36}$ Since these signaling pathways influence the corneal organization and play a role in regulation of extracellular matrix components, the genes encoding the core elements of the mentioned pathways were proposed as novel candidate genes for KTCN.

To assess the involvement of new genetic variants in KTCN etiology, we performed a further molecular investigation of corneas of Polish patients with KTCN, previously tested by RNA-Seq, using exome sequencing (ES) approach.

\section{Materials \& Methods}

\section{Patients}

All patients with KTCN underwent a complete ophthalmic evaluation in the Department of Ophthalmology, Medical University of Warsaw, Poland. The KTCN diagnosis was made based on the criteria previously described. ${ }^{37,33}$ The study protocol was approved by the Institutional Review Board at Poznan University of Medical Sciences (453/14; 755/19). All individuals provided informed consent after the possible consequences of the study were explained, in accordance with the Declaration of Helsinki.

\section{Material collection and DNA extraction}

The pairs of whole corneal tissues and blood samples were obtained from previously evaluated five $\mathrm{KTCN}$ patients $(\mathrm{KC15}, \mathrm{KC16}, \mathrm{KC17}, \mathrm{KC1} \text {, and } \mathrm{KC19})^{33}$ undergoing a penetrating keratoplasty procedure. The clinical characteristics of these patients are presented in Supplemental Table 1. Genomic DNA samples from the corneas were extracted using the Cells 
93 and Tissue DNA Isolation Kit (Norgen Biotek, Thorold, ON, Canada) according to the

94 manufacturer's protocol. Genomic DNA samples were isolated from the blood lymphocytes

95 using Gentra Puregene Blood Kit (Qiagen, Hilden, Germany), as previously described. ${ }^{37}$

96 Exome sequencing (ES)

97 This pilot ES study was conducted with $50 \mathrm{ng}$ of genomic DNA of whole corneal tissues (KC15,

$98 \mathrm{KC16}, \mathrm{KC17}, \mathrm{KC1}$, and $\mathrm{KC19}$ ) using the SureSelectQXT Reagent Kit combined with the

99 SureSelectXT Human All Exon V5 (Agilent Technologies, Cedar Creek, TX, USA) according to

100 manufacturer's instruction. Prepared libraries were paired-end sequenced $(2 \times 100 \mathrm{bp})$ on an

101 Illumina HiSeq1500 (Illumina, San Diego, CA, USA). For each cornea sample, $>80 \mathrm{mln}$ read

102 pairs were generated resulted in $>100 x$ of mean coverage. Sequence readouts were initially

103 analyzed with bcl2fastq software to generate reads in fastq format. These reads were mapped

104 against a human genome reference sequence (GRCh37) using the Burrows-Wheeler Alignment

105 (BWA), which was followed by BAM post-processing and variant calling using HaplotypeCaller 106 and the GATK suite. ${ }^{38}$ Finally, ANNOVAR ${ }^{39}$ was used to annotate relevant information about

107 gene names, predicted variant pathogenicity, reference allele frequencies and metadata from

108 external resources, and then to add these to the variant call format (VCF) file.

109

110

111

112

113

114

\section{Variants selection}

Sequence variants identified in KTCN corneas were filtered in a step-wise manner to exclude synonymous variants and variants with minor allele frequency (MAF) greater than 0.01 in our internal exome database (DMG) consisting of 3,000 Polish individuals, ExAC Browser (http://exac.broadinstitute.org/), GnomAD database (https://gnomad.broadinstitute.org/), and the 1000 Genomes Project (http://www.1000genomes.org). Moreover, variants predicted as neutral 
115 by MutationTaster ${ }^{40}$, PolyPhen- $2^{41}$, LRT $^{42}$, and SIFT ${ }^{43}$ tools, were filtered out. The additional

116 exclusion criterion was negative conservation scores in $\mathrm{PhyloP}^{44}$ analysis.

\section{Sanger sequencing}

118 To confirm variants detected by ES in five KTCN corneas, Sanger sequencing of DNA in 119 matching blood samples was performed. Briefly, fragments of genes containing particular 120 variants were amplified using Taq DNA Polymerase (Thermo Scientific, San Jose, CA, USA).

121 Following purifications with the use of FastAP Thermosensitive Alkaline Phosphatase and

122 Exonuclease I (Thermo Scientific), the amplicons were sequenced using BigDye Terminator

123 v3.1 Cycle Sequencing Kit (Applied Biosystems Inc., Foster City, CA, USA). Samples were 124 analyzed on an ABI Prism 3730xl genetic analyzer (Applied Biosystems Inc.). Sequences were 125 assembled using Sequencher 5.0. Software (GeneCodes Corporation, Ann Arbor, MI, USA).

\section{Pathway overrepresentation analysis}

127 The overrepresentation analysis of molecular pathways among genes with identified sequence

128 variants, which met the filtering criteria, was performed using the ConsensusPathDB tool. ${ }^{45}$ Only

129 pathways with $p$-value $\leq 0.01$ and sharing at least two genes with our gene set were analyzed.

\section{Ethnically-matched control datasets}

131 For comparison purposes, the datasets comprising ES data of five randomly selected Polish

132 individuals without ocular abnormalities and five Polish patients with high myopia (HM) were

133 used. Variants from control ES data were selected using the same filtering criteria as we used for

134 the KTCN study, followed by enrichment analyses.

\section{Expression analysis}

136 Expression levels of selected genes from overrepresented pathways, given in the gene-level

137 transcripts per million (TPM), were obtained from the RNA-Seq study, which has been 
138 previously performed in the same corneal material. ${ }^{33}$ The expression values of the particular

139 genes were compared between corneal samples in which variant(s) was identified and in samples

140 without the analyzed variant.

\section{Results}

142 This pilot ES screening of KTCN corneas revealed 117 potentially relevant variants with MAF $\leq$ 1430.01 in our internal DMG cohort and public databases and fulfilling all of the remaining filtering 144 criteria (Supplemental Table 2). Genes with identified rare nucleotide variants were enriched in 14514 molecular pathways, including Wnt signaling (VANGL1, WNT1, PPP3CC, LRP6, FZD2) and 146 focal adhesion (BIRC2, PAK6, COL4A4, PPP1R12A, PTK6) pathways (Table 1). The analysis of

147 functional interactions between genes from overrepresented molecular pathways allowed for 148 further narrowing the number of candidate KTCN variants (Table 2).

149 Sanger sequencing performed in blood samples derived from the five studied individuals 150 confirmed all variants identified with the use of ES in matched corneas.

The overrepresentation analysis performed among genes filtered in a step-wise manner in control datasets revealed an enrichment in 16 (i.e. O-linked glycosylation, C-type lectin

153 receptors, termination of O-glycan biosynthesis, nucleotide-binding oligomerization domain) and 154 nine (i.e. cargo recognition for clathrin-mediated endocytosis VLDL clearance, glyoxylate and 155 dicarboxylate metabolism, statin pathway DNA damage recognition in GG-NER) pathways in 156 HM patients and individuals without ocular disease, respectively. No genes involved in pathways 157 enriched in KTCN corneas were overrepresented in our control sample sets containing randomly 158 selected Polish individuals without ocular abnormalities $(\mathrm{n}=5)$ and Polish patients with HM $159(n=5)$. 
particular gene variants and in KTCN individuals without the analyzed gene variation were

162

163

164

165

166

167

168

169

170

171

172

173

174

175

176

177

178 calculated based on our previous RNA-Seq study data. ${ }^{33}$ The expression values of the genes varied between patients with particular variants and the mean expression values of the same genes in the KTCN individuals without these variants. The expression profile of genes from overrepresented pathways in KTCN corneas is presented in Table 3.

The data were deposited in the freely accessible ClinVar database (submission ID: SUB3758236 and SUB4926771).

\section{Discussion}

The advent of next generation DNA sequencing methods has greatly improved the ability to detect genetic variations. ${ }^{46}$ However, $\mathrm{KTCN}$, like many other ophthalmic diseases, displays genetic heterogeneity hindering identification of a factor unambiguously influencing its development. ${ }^{1,47}$

The results of this pilot ES study of corneas obtained from five KTCN patients undergoing penetrating keratoplasty revealed various rare possibly pathogenic variants in genes that were overrepresented in several molecular pathways, including the Wnt signaling and focal adhesion. Interestingly, these pathways have been proposed as involved in KTCN etiology based on our previous RNA-Seq study, performed in experimental material derived from the same KTCN patients, as well as other experiments. ${ }^{33-36}$ In addition, each of five patients had at least one variant in genes from these particular pathways. Among genes from overrepresentation gene sets were LRP6, FZD2, COL4A4, and WNT1.

Ocular cells, including corneal epithelial stem cells, express components of the Wnt/ $\beta$ catenin signaling pathway during eye development. ${ }^{48}$ The LRP6 gene encodes the low-density 
183 lipoprotein receptor-related protein, which is a component of a Wnt receptor complex. This 184 complex is involved in Wnt ligands binding resulting in the nuclear translocation of $\beta$-catenin 185 and regulation of the transcription of target genes. ${ }^{49}$ Knock-out of $\beta$-catenin or its both co186 receptors ( Lrp5 and Lrp6) in mouse corneal stromal cells resulted in premature stratification of 187 the corneal epithelium, suggesting these genes play a role in the regulation of corneal 188 morphogenesis. ${ }^{49}$ In this study, the expression of LRP6 in the cornea of the carrier of variant c.4822C $>\mathrm{T}(\mathrm{KC} 17)$ was unchanged compared to the other four KTCN individuals. However, since this variant was identified in $\mathrm{KC} 17$ patient in combination with another variant in the gene of Wnt signaling pathway $(P P P 3 C C)$, we suggest that this variant might be a part of the specific combination of KTCN variants and its identification is not incidental. including the developing eye.${ }^{50}$ Interestingly, a significant increase of mRNA and protein expression of secreted frizzled-related protein 1 (SFRP1), which is a Wnt antagonist, has been detected in KTCN corneal epithelium and corneal buttons. ${ }^{51,52}$ In contrast, tear SFRP1 level has been significantly decreased in KTCN patients compared to control individuals. ${ }^{53}$ The c.1238G $>$ A variant in FZD2 was identified in the cornea of $\mathrm{KC} 16$ patient in combination with c.41C $>$ T variant in $V A N G L 1$ (Wnt signaling) and c. $2347 \mathrm{G}>\mathrm{A}$ variant in COL4A4 (focal adhesion). It is known that collagens are major components of human corneas, and the thinning of corneal stroma in KTCN may be the final effect of a disorganized collagen lamellae arrangement. ${ }^{54,55}$ The COL4A4 gene is expressed in human cornea ${ }^{33}$ and based on several studies

205 it has been reported as a candidate gene for KTCN. ${ }^{56-59}$ However, genetic analyses of this gene 
206 in different populations have given ambiguous results about the role of COL $4 A 4$ in KTCN

207 development. ${ }^{56-59}$ While there was no difference in expression of COL4A4 and VANGL1 in the

208 patient's cornea, the expression value of FZD2 in the patient $\mathrm{KC1} 16$ was lower compared to the

209 expression observed in the other four KTCN individuals. However, further research should be

210 performed to interpret the obtained data.

211 The combination of variants in WNT1 and PTK6 was revealed in the patient KC15. PTK6

212 encodes tyrosine kinase 6 protein, which is involved in focal adhesion, as well as in GTPases and

213 MAP kinases regulation. Signaling by PTK6 is implicated in controlling the differentiation of

214 normal epithelium and tumor growth. ${ }^{60}$ However, there is no report about the role of PTK6 in the

215 maintenance of corneal epithelium.

216 Also, in KC18 and KC19 patients, variants in other elements (BIRC2 and PAK6 with

217 PPPIR12A, respectively) of Wnt signaling and/or focal adhesion pathways were observed.

218 These results further suggest that the alteration of genes regulating these signaling pathways

219 might be an important risk factor for KTCN.

220

To determine the likelihood of seeing the overrepresentation of genes from Wnt signaling and focal adhesion in KTCN samples by chance, we performed analyses of exome data of five randomly selected Polish individuals without eye disease, as well as five Polish individuals with HM. Variants from control ES data were selected using the same filtering criteria as we used for the KTCN study, followed by enrichment analyses. No genes involved in Wnt signaling or focal adhesion pathways were overrepresented in our control sample sets, confirming that enrichment of variants in genes from these pathways in KTCN individuals was not incidental.

The small sample size disabled a reliable statistical analysis, and due to this limitation, the general conclusions could not be made. However, the results of this pilot study gave additional 
229 insight into the role of the Wnt signaling and/or focal adhesion pathways in KTCN development

230 and showed the possible indications for further KTCN research. The identification of rare

231 variants in different genes further supports the heterogeneity of KTCN with multiple genes

232 underlying its pathogenesis. ${ }^{1,12-15}$

\section{Conclusions}

234 Summarizing, this first pilot ES profiling of human KTCN corneas indicates that the

235 accumulation of variants in several genes from Wnt signaling and/or focal adhesion pathways

236 might cause the phenotypic effect and further explain the involvement of these pathways in

237 KTCN. Moreover, it also supports the hypothesis about the complex basis of KTCN. Since five

238 patients were evaluated, the role of variants in genes of these overrepresented pathways in

239 KTCN etiology should be further elucidated in a larger group of patients using high throughput

240 methods.

242 References

243 1. Karolak JA, Gajecka M. Genomic strategies to understand causes of keratoconus. Mol

244 Genet Genomics. 2017;292:251-269. doi:10.1007/s00438-016-1283-z.

245 2. Rabinowitz YS. Keratoconus. Surv Ophthalmol. 1998;42:297-319.

246 3. Sarezky D, Orlin SE, Pan W, et al. Trends in Corneal Transplantation in Keratoconus.

247 Cornea. 2017;36:131-137. doi:10.1097/ICO.0000000000001083.

248 4. Vazirani J, Basu S. Keratoconus: current perspectives. Clin Ophthalmol. 2013;7:2019-2030.

249 doi:10.2147/OPTH.S50119. 
250 5. Pearson AR, Soneji B, Sarvananthan N, et al. Does ethnic origin influence the incidence or

251 severity of keratoconus? Eye (Lond). 2000;14 ( Pt 4):625-628. doi:10.1038/eye.2000.154.

252 6. Georgiou T, Funnell CL, Cassels-Brown A, et al. Influence of ethnic origin on the 253 incidence of keratoconus and associated atopic disease in Asians and white patients. Eye 254 (Lond). 2004;18:379-383. doi:10.1038/sj.eye.6700652.

255 7. Kok YO, Tan GFL, Loon SC. Review: keratoconus in Asia. Cornea. 2012;31:581-593. 256 doi:10.1097/ICO.0b013e31820cd61d.

257 8. Patel D, McGhee C. Understanding keratoconus: what have we learned from the New 258 Zealand perspective? Clin Exp Optom. 2013;96:183-187. doi:10.1111/cxo.12006.

9. Gokhale NS. Epidemiology of keratoconus. Indian J Ophthalmol. 2013;61:382-383.

10. Godefrooij DA, de Wit GA, Uiterwaal CS, et al. Age-specific incidence and prevalence of keratoconus: a nationwide registration study. Am J Ophthalmol. December 2016. doi:10.1016/j.ajo.2016.12.015.

11. Hashemi H, Heydarian S, Hooshmand E, et al. The Prevalence and Risk Factors for Keratoconus: A Systematic Review and Meta-Analysis. Cornea. September 2019. doi:10.1097/ICO.0000000000002150. stand? J Ophthalmol. 2014;2014:641708. doi:10.1155/2014/641708. 
269 13. Bykhovskaya Y, Margines B, Rabinowitz YS. Genetics in Keratoconus: where are we? Eye $270 \quad$ Vis (Lond). 2016;3:16. doi:10.1186/s40662-016-0047-5.

271 14. Mas Tur V, MacGregor C, Jayaswal R, et al. A review of keratoconus: Diagnosis,

272 pathophysiology, and genetics. Surv Ophthalmol. 2017;62:770-783.

273 doi:10.1016/j.survophthal.2017.06.009.

15. Valgaeren H, Koppen C, Van Camp G. A new perspective on the genetics of keratoconus: why have we not been more successful? Ophthalmic Genet. 2018;39:158-174. doi:10.1080/13816810.2017.1393831.

16. Loukovitis E, Sfakianakis K, Syrmakesi P, et al. Genetic Aspects of Keratoconus: A Literature Review Exploring Potential Genetic Contributions and Possible Genetic Relationships with Comorbidities. Ophthalmol Ther. 2018;7:263-292. doi:10.1007/s40123-

17. Tang YG, Rabinowitz YS, Taylor KD, et al. Genomewide linkage scan in a multigeneration Caucasian pedigree identifies a novel locus for keratoconus on chromosome 5q14.3-q21.1. Genet Med. 2005;7:397-405. doi:10.109701.GIM.0000170772.41860.54.

18. Li X, Rabinowitz YS, Tang YG, et al. Two-stage genome-wide linkage scan in keratoconus sib pair families. Invest Ophthalmol Vis Sci. 2006;47:3791-3795. doi:10.1167/iovs.06-0214.

19. Bisceglia L, De Bonis P, Pizzicoli C, et al. Linkage analysis in keratoconus: replication of locus 5q21.2 and identification of other suggestive Loci. Invest Ophthalmol Vis Sci. 2009;50:1081-1086. doi:10.1167/iovs.08-2382. 
289 20. Rosenfeld JA, Drautz JM, Clericuzio CL, et al. Deletions and duplications of

290 developmental pathway genes in 5q31 contribute to abnormal phenotypes. Am J Med Genet

291 A. 2011;155A:1906-1916. doi:10.1002/ajmg.a.34100.

292

293

294

295

296

297

298

299

300

301

302

303

304

305

306

307

308

309

21. Bykhovskaya Y, Li X, Taylor KD, et al. Linkage Analysis of High-density SNPs Confirms Keratoconus Locus at 5q Chromosomal Region. Ophthalmic Genet. 2016;37:109-110. doi: $10.3109 / 13816810.2014 .889172$.

22. Li X, Bykhovskaya Y, Haritunians T, et al. A genome-wide association study identifies a potential novel gene locus for keratoconus, one of the commonest causes for corneal transplantation in developed countries. Hum Mol Genet. 2012;21:421-429. doi:10.1093/hmg/ddr460.

23. Burdon KP, Macgregor S, Bykhovskaya Y, et al. Association of polymorphisms in the hepatocyte growth factor gene promoter with keratoconus. Invest Ophthalmol Vis Sci. 2011;52:8514-8519. doi:10.1167/iovs.11-8261.

24. Hoehn R, Zeller T, Verhoeven VJM, et al. Population-based meta-analysis in Caucasians confirms association with COL5A1 and ZNF469 but not COL8A2 with central corneal thickness. Hum Genet. 2012;131:1783-1793. doi:10.1007/s00439-012-1201-3.

25. Lu Y, Vitart V, Burdon KP, et al. Genome-wide association analyses identify multiple loci associated with central corneal thickness and keratoconus. Nat Genet. 2013;45:155-163. doi:10.1038/ng.2506.

26. Héon E, Greenberg A, Kopp KK, et al. VSX1: a gene for posterior polymorphous dystrophy and keratoconus. Hum Mol Genet. 2002;11:1029-1036. 
310 27. Karolak JA, Rydzanicz M, Ginter-Matuszewska B, et al. Variant c.2262A >C in DOCK9

311 Leads to Exon Skipping in Keratoconus Family. Invest Ophthalmol Vis Sci. 2015;56:7687-

312 7690. doi:10.1167/iovs.15-17538.

313

314
28. Udar N, Atilano SR, Brown DJ, et al. SOD1: a candidate gene for keratoconus. Invest Ophthalmol Vis Sci. 2006;47:3345-3351. doi:10.1167/iovs.05-1500.

29. Lucas SEM, Zhou T, Blackburn NB, et al. Rare, potentially pathogenic variants in 21 keratoconus candidate genes are not enriched in cases in a large Australian cohort of European descent. PLoS ONE. 2018;13:e0199178. doi:10.1371/journal.pone.0199178.

30. Karolak JA, Gambin T, Pitarque JA, et al. Variants in SKP1, PROB1, and IL17B genes at keratoconus 5q31.1-q35.3 susceptibility locus identified by whole-exome sequencing. Eur $J$ Hum Genet. 2017;25:73-78. doi:10.1038/ejhg.2016.130.

31. Khaled ML, Bykhovskaya Y, Gu C, et al. PPIP5K2 and PCSK1 are Candidate Genetic Contributors to Familial Keratoconus. Sci Rep. 2019;9:19406. doi:10.1038/s41598-01955866-5.

32. Magalhães $\mathrm{O}$ de A, Kowalski TW, Wachholz GE, et al. Whole-exome sequencing in familial keratoconus: the challenges of a genetically complex disorder. Arq Bras Oftalmol. 2019;82:453-459. doi:10.5935/0004-2749.20190087.

33. Kabza M, Karolak JA, Rydzanicz M, et al. Collagen synthesis disruption and downregulation of core elements of TGF- $\beta$, Hippo, and Wnt pathways in keratoconus corneas. Eur J Hum Genet. 2017;25:582-590. doi:10.1038/ejhg.2017.4. 
330 34. Khaled ML, Bykhovskaya Y, Yablonski SER, et al. Differential Expression of Coding and 331 Long Noncoding RNAs in Keratoconus-Affected Corneas. Invest Ophthalmol Vis Sci. 2018;59:2717-2728. doi:10.1167/iovs.18-24267.

333

334

335

336

337

338

339

340

341

342

343

344

345

346

347

348

349

35. You J, Corley SM, Wen L, et al. RNA-Seq analysis and comparison of corneal epithelium in keratoconus and myopia patients. Sci Rep. 2018;8:389. doi:10.1038/s41598-017-18480$\mathrm{X}$.

36. Sharif R, Khaled ML, McKay TB, et al. Transcriptional profiling of corneal stromal cells derived from patients with keratoconus. Sci Rep. 2019;9:12567. doi:10.1038/s41598-01948983-8.

37. Karolak JA, Gambin T, Rydzanicz M, et al. Evidence against ZNF469 being causative for keratoconus in Polish patients. Acta Ophthalmol. 2016;94:289-294. doi:10.1111/aos.12968.

38. McKenna A, Hanna M, Banks E, et al. The Genome Analysis Toolkit: a MapReduce framework for analyzing next-generation DNA sequencing data. Genome Res. 2010;20:1297-1303. doi:10.1101/gr.107524.110.

39. Wang K, Li M, Hakonarson H. ANNOVAR: functional annotation of genetic variants from high-throughput sequencing data. Nucleic Acids Res. 2010;38:e164. doi:10.1093/nar/gkq603.

40. Schwarz JM, Rödelsperger C, Schuelke M, et al. MutationTaster evaluates disease-causing potential of sequence alterations. Nat Methods. 2010;7:575-576. doi:10.1038/nmeth0810575. 
350 41. Adzhubei I, Jordan DM, Sunyaev SR. Predicting functional effect of human missense

351 mutations using PolyPhen-2. Curr Protoc Hum Genet. 2013; Chapter 7:Unit7.20.

352 doi:10.1002/0471142905.hg0720s76.

353

354

42. Chun S, Fay JC. Identification of deleterious mutations within three human genomes. Genome Res. 2009;19:1553-1561. doi:10.1101/gr.092619.109.

43. Kumar P, Henikoff S, Ng PC. Predicting the effects of coding non-synonymous variants on protein function using the SIFT algorithm. Nat Protoc. 2009;4:1073-1081. doi:10.1038/nprot.2009.86.

44. Pollard KS, Hubisz MJ, Rosenbloom KR, et al. Detection of nonneutral substitution rates on mammalian phylogenies. Genome Res. 2010;20:110-121. doi:10.1101/gr.097857.109.

45. Kamburov A, Stelzl U, Lehrach H, et al. The ConsensusPathDB interaction database: 2013 update. Nucleic Acids Res. 2013;41:D793-800. doi:10.1093/nar/gks1055.

46. Koboldt DC, Steinberg KM, Larson DE, et al. The next-generation sequencing revolution and its impact on genomics. Cell. 2013;155:27-38. doi:10.1016/j.cell.2013.09.006.

47. Stone EM, Braun TA, Russell SR, et al. Missense variations in the fibulin 5 gene and agerelated macular degeneration. $N$ Engl J Med. 2004;351:346-353. doi:10.1056/NEJMoa040833.

48. Nakatsu MN, Ding Z, Ng MY, et al. Wnt/ $\beta$-catenin signaling regulates proliferation of human cornea epithelial stem/progenitor cells. Invest Ophthalmol Vis Sci. 2011;52:47344741. doi:10.1167/iovs.10-6486. 
370

371

372

373

374

375

376

377

378

379

380

381

382

49. Zhang Y, Yeh L-K, Zhang S, et al. Wnt/ $\beta$-catenin signaling modulates corneal epithelium stratification via inhibition of Bmp4 during mouse development. Development. 2015;142:3383-3393. doi:10.1242/dev.125393.

50. Deardorff MA, Klein PS. Xenopus frizzled-2 is expressed highly in the developing eye, otic vesicle and somites. Mech Dev. 1999;87:229-233.

51. Sutton G, Madigan M, Roufas A, et al. Secreted frizzled-related protein 1 (SFRP1) is highly upregulated in keratoconus epithelium: a novel finding highlighting a new potential focus for keratoconus research and treatment. Clin Experiment Ophthalmol. 2010;38:43-48. doi:10.1111/j.1442-9071.2009.02216.x.

52. You J, Wen L, Roufas A, et al. Expression of SFRP Family Proteins in Human Keratoconus Corneas. PLoS ONE. 2013;8:e66770. doi:10.1371/journal.pone.0066770.

53. You J, Hodge C, Wen L, et al. Tear levels of SFRP1 are significantly reduced in keratoconus patients. Mol Vis. 2013;19:509-xxx.

54. Meek KM, Tuft SJ, Huang Y, et al. Changes in collagen orientation and distribution in keratoconus corneas. Invest Ophthalmol Vis Sci. 2005;46:1948-1956. doi:10.1167/iovs.041253.

55. Mathew JH, Goosey JD, Söderberg PG, et al. Lamellar changes in the keratoconic cornea. Acta Ophthalmol. 2015;93:767-773. doi:10.1111/aos.12811.

56. Stabuc-Silih M, Ravnik-Glavac M, Glavac D, et al. Polymorphisms in COL4A3 and COL4A4 genes associated with keratoconus. Mol Vis. 2009;15:2848-2860. 
390 57. Wang Y, Jin T, Zhang X, et al. Common single nucleotide polymorphisms and keratoconus 391 in the Han Chinese population. Ophthalmic Genet. 2013;34:160-166.

392 doi:10.3109/13816810.2012.743569.

393

394

395

396

397

398

399

400

401

402
58. Kokolakis NS, Gazouli M, Chatziralli IP, et al. Polymorphism analysis of COL4A3 and COL4A4 genes in Greek patients with keratoconus. Ophthalmic Genet. 2014;35:226-228. doi:10.3109/13816810.2014.946055.

59. Saravani R, Hasanian-Langroudi F, Validad M-H, et al. Evaluation of possible relationship between COL4A4 gene polymorphisms and risk of keratoconus. Cornea. 2015;34:318-322. doi:10.1097/ICO.0000000000000356.

60. Brauer PM, Tyner AL. Building a better understanding of the intracellular tyrosine kinase PTK6 - BRK by BRK. Biochim Biophys Acta. 2010;1806:66-73. doi:10.1016/j.bbcan.2010.02.003. 


\section{Table $\mathbf{1}$ (on next page)}

Top pathways overrepresented across the genes with identified sequence variants in KTCN corneas detected by ConsensusPathDB server ( $p$-value cutoff 0.01 ).

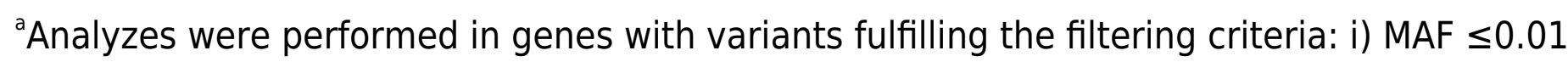
in our internal database, the ExAC Browser, GnomAD database, and the 1000 Genomes Project Variants; ii) positive conservation scores in PhyloP; iii) pathogenic/damaging in MutationTaster, PolyPhen2, LRT, and SIFT tools. 
Table 1. Top pathways overrepresented across the genes with identified sequence variants in KTCN corneas detected by 2 ConsensusPathDB server ( $\mathrm{p}$-value cutoff 0.01 ).

\begin{tabular}{llll}
\hline Pathway Name & Source & Genes $^{\text {a }}$ & p-value \\
\hline $\begin{array}{l}\text { Disassembly of the destruction complex and recruitment of } \\
\text { AXIN to the membrane }\end{array}$ & Reactome & FZD2, LRP6, WNT1 & 0.000873181 \\
\hline Wnt Signaling & Wikipathways & VANGL1, WNT1, PPP3CC, LRP6, FZD2 & 0.000893629 \\
\hline Type I hemidesmosome assembly & Reactome & PLEC, COL17A1 & 0.001477943 \\
\hline Wnt Signaling in Kidney Disease & Wikipathways & WNT1, LRP6, FZD2 & 0.001788119 \\
\hline Wnt signaling pathway - Homo sapiens (human) & KEGG & VANGL1, WNT1, PPP3CC, LRP6, FZD2 & 0.002830193 \\
\hline RAB GEFs exchange GTP for GDP on RABs & Reactome & $A N K R D 27$, DENND1A, RABGEF1, DENND4C & 0.002959219 \\
\hline The activation of arylsulfatases & Reactome & $A R S D$, ARSB & 0.003148218 \\
\hline Epithelial to mesenchymal transition in colorectal cancer & Wikipathways & WNT1, COL4A4, LRP6, FZD2, MEF2D & 0.003952926 \\
\hline cGMP-PKG signaling pathway - Homo sapiens (human) & KEGG & $A T P 2 A 1$, PIK3CG, PPP1R12A, PPP3CC, MEF2D & 0.004277236 \\
\hline MicroRNAs in cardiomyocyte hypertrophy & Wikipathways & HDAC9, FZD2, LRP6, PIK3CG & 0.004456905 \\
\hline Wnt-beta-catenin Signaling Pathway in Leukemia & Wikipathways & WNT1, LRP6 & 0.008974682 \\
\hline Rab regulation of trafficking & Reactome & $A N K R D 27$, DENND1A, RABGEF1, DENND4C & 0.009329868 \\
\hline Focal Adhesion & Wikipathways & BIRC2, PAK6, COL4A4, PPP1R12A, PTK6 & 0.009582658 \\
\hline Regulation of RAS by GAPs & Reactome & NF1, SPRED2 & 0.009787951 \\
\hline
\end{tabular}

3 aAnalyzes were performed in genes with variants fulfilling the filtering criteria: i) MAF $\leq 0.01$ in our internal database, the ExAC 4 Browser, GnomAD database, and the 1000 Genomes Project Variants; 5 pathogenic/damaging in MutationTaster, PolyPhen2, LRT, and SIFT tools. 


\section{Table 2 (on next page)}

The list of nonsynonymous sequence variants in genes from Wnt signaling and focal adhesion pathways, identified in KTCN corneas in ES

${ }^{\mathrm{a} H u m a n}$ Genome Browser - hg19 assembly (GRCh37) ${ }^{\mathrm{b}}$ Minor allele frequency based on internal control exome database covering of 3000 Polish individuals; Department of Medical

Genetics (DMG), Medical University of Warsaw, Warsaw, Poland; 'Minor allele frequency based on GnomAD database v2.1.2; ${ }^{\mathrm{d}} \mathrm{NCBI}$ dbSNP Build $151 ;{ }^{\mathrm{e}}$ Not available 
Table 2. The list of nonsynonymous sequence variants in genes from Wnt signaling and focal adhesion pathways, identified in KTCN corneas in ES

\begin{tabular}{|c|c|c|c|c|c|c|c|c|}
\hline Identifier & Gene & Acession Number & Position $^{a}$ & cDNA & Protein & $\mathbf{D M G}^{\mathbf{b}}$ & GnomAD $^{\mathbf{c}}$ & Rs_id ${ }^{d}$ \\
\hline \multirow{2}{*}{$\mathrm{KC} 15$} & WNT1 & NM_005430.3 & $\operatorname{chr} 12: 49375373 \mathrm{G}>\mathrm{T}$ & c. $1063 \mathrm{G}>\mathrm{T}$ & p.(V355F) & 0 & $\mathrm{NA}^{\mathrm{e}}$ & rs387907358 \\
\hline & PTK6 & NM_005975.3 & $\operatorname{chr} 20: 62161533 \mathrm{G}>\mathrm{T}$ & c. $1066 \mathrm{C}>\mathrm{A}$ & p.(P356T) & 0 & NA & NA \\
\hline \multirow{3}{*}{$\mathrm{KC} 16$} & FZD2 & NM_001466.3 & $\operatorname{chr} 17: 42636294 \mathrm{G}>\mathrm{A}$ & c. $1238 \mathrm{G}>\mathrm{A}$ & p.(R413Q) & 0 & 0.00002396 & rs758351214 \\
\hline & $V A N G L 1$ & NM_001172411.1 & $\operatorname{chr} 1: 116194075 \mathrm{C}>\mathrm{T}$ & c. $41 \mathrm{C}>\mathrm{T}$ & p.(S14L) & 0 & NA & NA \\
\hline & COL4A4 & NM_000092.4 & chr2:227924157C $>\mathrm{T}$ & c. $2347 \mathrm{G}>\mathrm{A}$ & p.(G783R) & 0.000333 & NA & rs 1202230056 \\
\hline \multirow{2}{*}{$\mathrm{KC} 17$} & $L R P 6$ & NM_002336.2 & chr12:12274080G $>$ A & $\mathrm{c} .4822 \mathrm{C}>\mathrm{T}$ & p.(P1608S) & 0 & NA & NA \\
\hline & PPP3CC & NM_001243975.1 & chr8:22389795T $>C$ & c. $1199 \mathrm{~T}>\mathrm{C}$ & p.(M400T) & 0 & NA & NA \\
\hline $\mathrm{KC} 18$ & $B I R C 2$ & NM_001166.4 & chr11:102248388A>G & c. $1528 \mathrm{~A}>\mathrm{G}$ & p.(I510V) & 0.001 & 0.00002148 & rs749829698 \\
\hline \multirow{2}{*}{ KC19 } & PPP1R12A & NM_001244992.1 & $\operatorname{chr} 12: 80266702 \mathrm{~T}>\mathrm{C}$ & c. $254 \mathrm{~A}>\mathrm{G}$ & p.(N85S) & 0.000667 & 0.00002422 & rs370959842 \\
\hline & PAK6 & NM_001276717.1 & chr15:40566454C $>$ A & c. $1855 \mathrm{C}>\mathrm{A}$ & p.(P619T) & 0 & NA & NA \\
\hline
\end{tabular}

4

5 aHuman Genome Browser - hg19 assembly (GRCh37)

6 bMinor allele frequency based on internal control exome database covering of 3000 Polish individuals; Department of Medical

7 Genetics (DMG), Medical University of Warsaw, Warsaw, Poland

8 cMinor allele frequency based on GnomAD database v2.1.2

9 dNCBI dbSNP Build 151

10 eNot available 


\section{Table 3(on next page)}

The expression of genes with rare variants identified in ES analysis.

Grey color indicates the expression level in samples in which variant was present, and white color indicates the expression level of genes in samples without presence of the analyzed variant. 
1 Table 3. The expression of genes with rare variants identified in ES analysis.

\begin{tabular}{|c|c|c|c|c|c|c|c|}
\hline Pathway & $\begin{array}{l}\text { Gene } \\
\text { symbol/ } \\
\text { Variant }\end{array}$ & KC_15 & $K C_{-} 16$ & KC_17 & $\mathrm{KC}_{-} 18$ & KC_19 & $\begin{array}{l}\text { Mean } \\
\text { expression }\end{array}$ \\
\hline Wnt Signaling & $\begin{array}{l}\text { WNT1 } \\
\text { c. } 1063 \mathrm{G}>\mathrm{T}\end{array}$ & 0.051 & 0.000 & 0.000 & 0.018 & 0.023 & 0.0103 \\
\hline Focal Adhesion & $\begin{array}{l}P T K 6 \\
\text { c. } 1066 \mathrm{C}>\mathrm{A}\end{array}$ & 27.705 & 31.413 & 22.991 & 25.809 & 26.031 & 26.5610 \\
\hline Wnt Signaling & $\begin{array}{l}F Z D 2 \\
\text { c. } 1238 \mathrm{G}>\mathrm{A}\end{array}$ & 1.254 & 0.151 & 2.988 & 0.453 & 0.544 & 1.3098 \\
\hline Wnt Signaling & $\begin{array}{l}V A N G L 1 \\
\text { c. } 41 \mathrm{C}>\mathrm{T}\end{array}$ & 4.655 & 3.581 & 4.129 & 3.132 & 5.074 & 4.2475 \\
\hline Focal Adhesion & $\begin{array}{l}C O L 4 A 4 \\
\text { c. } 2347 \mathrm{G}>\mathrm{A}\end{array}$ & 8.802 & 8.487 & 15.100 & 5.863 & 7.398 & 9.2908 \\
\hline Wnt Signaling & $\begin{array}{l}L R P 6 \\
\text { c. } 4822 \mathrm{C}>\mathrm{T}\end{array}$ & 14.144 & 13.303 & 11.900 & 11.515 & 12.581 & 12.8858 \\
\hline Wnt Signaling & $\begin{array}{l}P P P 3 C C \\
\text { c. } 1199 \mathrm{~T}>\mathrm{C}\end{array}$ & 12.523 & 14.479 & 9.580 & 8.556 & 10.525 & 11.5208 \\
\hline Focal Adhesion & $\begin{array}{l}\text { BIRC2 } \\
\text { c. } 1528 \mathrm{~A}>\mathrm{G}\end{array}$ & 42.675 & 45.517 & 32.853 & 30.261 & 40.725 & 40.4425 \\
\hline Focal Adhesion & $\begin{array}{l}P P P 1 R 12 A \\
\text { c. } 254 \mathrm{~A}>\mathrm{G}\end{array}$ & 83.939 & 83.438 & 61.009 & 55.103 & 76.460 & 70.8723 \\
\hline Focal Adhesion & $\begin{array}{l}P A K 6 \\
\text { c. } 1855 \mathrm{C}>\mathrm{A}\end{array}$ & 8.818 & 7.144 & 5.704 & 4.970 & 6.011 & 6.6590 \\
\hline
\end{tabular}

2

3 Grey color indicates the expression level in samples in which variant was present, and white

4 color indicates the expression level of genes in samples without presence of the analyzed variant. 